\section{CRYSTAL STRUCTURE ANALYSES OF THERMOLYSIN IN COMPLEX WITH ITS INHIBITORS}

M. Senda ${ }^{1}$ T. Senda ${ }^{2}$ T. Ogi ${ }^{3}$ S. Kidokoro

National Inst. of Advanced Industrial Science and Technology (AIST)

Biological Information Research Center Aomi 2-41-6, Koto-Ku TOKYO 1350064 JAPAN

${ }^{1}$ Japan Biological Information Research Center, Japan Biological Infomatics Consortium ${ }^{2}$ Biological Information Research Center, National Institute of Advanced Industrial Science and Technology ${ }^{3}$ Department of Bioengineering, Nagaoka University of Technology

Thermolysin (TLN) is a thermostable metalloendpeptidase from the thermophilic bacterium Bacillus thermoproteolyticus. Kidokoro and coworkers have studied the binding mode of a series of inhibitors, namely benzyloxycarbonyl amino acids (Z-amino acids), to TLN through thermodynamic methods and computer simulations. In order to analyze these results on the basis of their three-dimensional structures, the crystal structures of TLN in complex with Z-amino-acid (Z-L-Glu, Z-D-Glu, Z-L-Asp, Z-D-Asp, Z-L-Thr, Z-D-Thr, Z-L-Ala and Z-D-Ala) have been determined at 1.6 to $1.9 \AA$ resolution. These crystal structures showed that the benzyl group and a carboxyl group of the inhibitor seem to be main contributors to the interaction between TLN and the inhibitors. The benzyl group of the inhibitors, in all cases, occupies the hydrophobic specificity pocket of TLN. The interaction between the zinc ion and a carboxyl group of the substrate, however, showed some variations. In the case of the L-form inhibitors, the main-chain carbonyl group of the inhibitors coordinates the zinc ion, resulting in essentially the same conformations of the bound L-form inhibitors. In the case of the D-form inhibitors, however, the coordination mode of the zinc ion was classified into two types, namely main-chain and side-chain types. Z-D-Glu and -Asp use the side-chain carboxyl group to coordinate the zinc ion. On the other hand, Z-DAla and -Thr, which do not have a carboxyl group in the side-chain, use the main-chain carboxyl group to coordinate the zinc ion. The conformations of the bound D-form inhibitors are substantially different between main- and sidechain type binding modes.

Keywords: HYDROLASE, METALLOPROTEINASE

\section{Acta Cryst. (2002). A58 (Supplement), C278}

\section{IS TRNA MIMICRY MANDATORY FOR TRANSLATION FACTORS?} A. Liljas S. Hansson O. Kristensen M. Laurberg D. Logan S. Sanyal M. Selmer X.-D. Su

Molecular Biophysics, Centre for Chemistry and Chemical Engineering, Lund University Box 124 LUND SE-221 00 SWEDEN

tRNA is the adaptor in protein synthesis. The ribosome has three sites for tRNA, the A-, P-, and E-sites. The tRNAs bridge between the ribosomal subunits with the decoding site and the mRNA on the 30S subunit and the peptidyl transfer site on the 50S subunit. Translation factors catalyze protein synthesis on the ribosome. We study elongation factors, primarily EF-G, RRF and SelB. SelB can incorporate Se-Cys into proteins as a response to a stop codon. The distinction between stop and Se-Cys is a specific secondary structure of the mRNA. We have determined the structure of the C-terminal four domains that identify the loop of the mRNA. The domains have the same folding motif. For EF-G we continue our investigations of different mutants and different crystal forms. The focus on resistance and sensitivity to the antibiotic fusidic acid and its parallel higher or lower affinity for GTP. The possibility that translation factors could mimic tRNA has been discussed for some time. The first example of tRNA mimicry was discovered when EF-G was found to have a shape closely similar to the complex between elongation factor Tu (EF-Tu), GTP and aminoacyl-tRNA. An even closer imitation of the tRNA shape is seen for the ribosome recycling factor (RRF). It is now evident that in some sense translation factors are either tRNA mimics, GTPases or both. In many cases this mimicry is related to function. However, for some proteins, such as SelB, there is no obvious functional reason for the tRNA mimicry.

Keywords: PROTEIN SYNTHESIS, TRANSLATION FACTORS, TRNA MIMICRY

Acta Cryst. (2002). A58 (Supplement), C278

CRYSTAL ENGINEERING YIELDS CRYSTALS OF CYCLOPHILIN D DIFFRACTING TO 1.5 A RESOLUTION

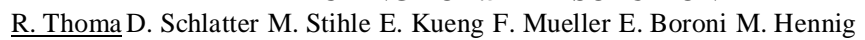
F. Hoffmann-La Roche Discovery Technologies Bldg. 065/505 BASEL CH4070 SWITZERLAND

Mitochondrial Cyclophilin D (CypD) belongs to a family of cyclosporin-A binding proteins, which catalyse rotation about prolyl peptide bonds. Structures of the three other types of mammalian cyclophilins (A, B and C) are known. A truncated version of CypD with the same length as CypA was expressed in Escherichia coli and purified to homogeneity. The protein shows a monodisperse size distribution in analytical ultracentrifugation, but crystallization trials gave no crystals. Protein engineering has become a powerful tool for influencing protein properties. Therefore, site-directed mutagenesis was used to obtain a variant of CypD suitable for crystallization and structure determination. A series of mutants was selected such that crystal contacts seen in the X-ray structures of CypA would be restored. None of these three mutants gave crystals. Because CypD is extremely soluble and has an unusually high isoelectric point (pI) of 10.2, a second series of mutations was introduced in order to lower the $\mathrm{pI}$ of CypD and to reduce the solubility of the protein. Lysine residues predicted to be on the molecular surface of CypD were changed to hydrophobic or negatively charged amino acids found in the homologous at the corresponding position. This approach lead to crystals in one out of five variants of single amino acid exchanges. The crystals of the mutant K133I yielded readily crystals diffracting to $1.5 \AA$ resolution. This encouraging result shows that surface mutations have a dramatic effect on the protein and are a powerful tool for the improvement of protein crystallization properties.

\title{
Keywords: CRYSTAL ENGINEERING
}

Acta Cryst. (2002). A58 (Supplement), C278

\section{STRUCTURAL STUDIES ON TRANSLATION INTIATION FACTOR 4E}

K. Tomoo ${ }^{1}$ X. Shen ${ }^{1}$ K. Okabe ${ }^{1}$ Y. Nozoe ${ }^{1}$ S. Fukuhara ${ }^{1}$ S. Morino ${ }^{1}$ T. Ishida ${ }^{1}$ T. Taniguchi ${ }^{2}$ M. Sasaki ${ }^{2}$ Y. Katsuya ${ }^{3}$ K. Kitamura ${ }^{4}$ H. Miyoshi ${ }^{5}$ M. Ishikawa K. Miura ${ }^{5}$

${ }^{1}$ Osaka University of Pharmaceutical Sciences Department of Physical Chemistry 4-20-1 Nasahara TAKATSUKI 569-1094 JAPAN ${ }^{2}$ Hyogo Institute for Aging Brain and Cognitive Disorders ${ }^{3}$ Hyogo Prefectural Institute of Industrial Research ${ }^{4}$ Research Center, Taisho Pharmaceutical Co., Ltd.

${ }^{5}$ Institute for Biomolecular Science, Gakushuin University

Translation initiation in eukaryotes requires a complex biochemical pathway catalyzed by a large number of initiation factors. The first step of the translation process involves binding of small ribosomal subunit to mRNA. Eukaryotic mRNA has a common cap structure at the 5-terminal end. Eukariotic initiation factor 4E (eIF4E) binds specifically to the mRNA cap structure and plays an important role in the beginning of protein synthesis. Elucidation of the interaction between eIF4E and the mRNA cap structure is therefore necessary to understand the initiation mechanism of protein synthesis. The full-length human eIF4E in complex with the cap analogues were crystallized by hanging drop vapor diffusion. Long needle shaped crystals were obtained after one week. X-ray diffraction data were collected up to $1.9 \square$ ð resolution using synchrotron radiation at BL-24XU in Spring-8. The X-ray structures of eIF4E complexed with mRNA cap analogs (m7GTP and m7GpppA) were determined by the molecular replacement method at $2.0 \AA$ resolution. We have observed the $\mathrm{C}$-terminal region shows the conformational flexibility depending on the structure of mRNA cap analog when compared eIF4E-m7GTP and -m7GpppA complex structures. These results indicate that the high flexibility of C-terminal loop region is very important for binding with capped mRNA.

Keywords: TRANSLATION INITIATION FACTOR CAP ANALOG 